\title{
MiR-425-5p accelerated the proliferation, migration, and invasion of ovarian cancer cells via targeting AFF4
}

Zhihui Wu' ${ }^{1}$ Jianlin $\mathrm{GuO}^{2}$, Ying Zhang ${ }^{3}$, Jianhua Liu ${ }^{4^{*}} \mathbb{D}$, Hongping $\mathrm{Ma}^{5^{*}}$ and Yurong Tang ${ }^{6^{*}}$

\begin{abstract}
Background: Accumulating data have established that microRNAs (miRNAs) play significant regulatory roles in the carcinogenesis and progression of ovarian cancer (OC). MiR-425-5p was reported to function in various tumors. However, the roles and underlying mechanism of miR-425-5p involvement in OC development and progression are unclear.

Methods: A comprehensive strategy of data mining, computational biology, and real-time polymerase chain reaction was employed to identify the involvement of miR-425-5p in OC progression. The effect of miR-425-5p on the proliferation, migration, and invasion of OC cells was determined using Cell Counting Kit-8, wound-healing, and Matrigel invasion assays, respectively. Luciferase assay was performed to evaluate the interactions between miR-425-5p and MAGI2-AS3 Or AFF4.
\end{abstract}

Results: miR-425-5p was significantly up-regulated in OC tissues and cells. The luciferase reporter assay revealed that miR-425-5p was negatively regulated by MAGI2-AS3. Silencing miR-425-5p inhibited the proliferation, migration, and invasion of OC cells in vitro. Bioinformatics analysis and luciferase reporter assay revealed that AFF4 was the target gene of miR-425-5p. Moreover, AFF4 expression was significantly decreased in OC and was closely related to the good prognosis of patients with OC. AFF4 overexpression inhibited the proliferation, migration, and invasion of OC cells in vitro. By contrast, silencing AFF4 promoted the proliferation, migration, and invasion of OC cells in vitro. Finally, AFF4 suppression rescued the inhibitory effect of silencing miR-425-5p on the proliferation, migration, and invasion of OC cells.

Conclusion: To the best our knowledge, this is the first study to demonstrate that miR-425-5p overexpression in $O C$ is negatively regulated by MAG/2-AS3. Moreover, miR-425-5p promotes the proliferation, migration, and invasion of OC cells by targeting AFF4, suggesting that miR-425-5p/AFF4 signaling pathway represented a novel therapeutic target for patients with OC.

Keywords: Ovarian cancer, miR-425-5p, AFF4, Proliferation, Invasion, Metastasis

\footnotetext{
*Correspondence: liuying4544@126.com; 335357208@qq.com; zxyyjyktyr@163.com

${ }^{4}$ Department of Clinical Laboratory, Beijing Rehabilitation Hospital, Capital Medical University, Beijing 100144, China

${ }^{5}$ Department of Clinical Laboratory, Children's Hospital of Xinjiang Uygur

Autonomous Region, Urumqi, Xinjiang 830054, Uygur Autonomous Region, China

${ }^{6}$ Laboratory Department of Shengli Oilfield Central Hospital, Dongying 257100, China

Full list of author information is available at the end of the article
}

\begin{abstract}
Introduction
Ovarian cancer $(\mathrm{OC})$ is one of the major malignancies of the female reproductive system, and it has the highest incidence rate among all gynecological malignancies worldwide. The high incidence is associated with the difficulties of efficient early detection of OC because of its asymptomatic early stage [1]. Despite the availability of surgery, chemotherapy, and radiotherapy, the 5-year
\end{abstract}


overall survival rate of $\mathrm{OC}$ was not improved in the past decades [2]. Therefore, it is urgent to examine the mechanism underlying $\mathrm{OC}$ development and to find new therapeutic targets to improve the clinical outcomes of patients with OC.

Given the advances in high-resolution microarray and massively parallel sequencing technology, emerging evidence indicates that microRNAs (miRNAs) play complex and extensive roles in the development and progression of various cancers [3]. Studies have reported the involvement of $m i R-425-5 p$ in various cancer processes, and it acts as a tumor suppressor or promoter $[4,5]$. However, the exact roles and underlying mechanism of $m i R-425-5 p$ function in $\mathrm{OC}$ are still unclear.

In this study, the results demonstrated that miR-425-5p was upregulated in $\mathrm{OC}$, and it was negatively regulated by MAGI2-AS3. Furthermore, AF4/FMR2 family member 4 (AFF4), as a tumor-suppressor gene in OC, was the new target of $m i R-425-5 p$. Finally, silencing $m i R-425-5 p$ suppressed the proliferation, migration, and invasion of OC by targeting upregulated AFF4.

\section{Materials and methods Data sources}

MAGI2-AS3 expression data in OC were acquired from the online databases of Gene Expression Profiling Interactive Analysis (GEPIA, http://gepia.cancer-pku.cn/) and Gene Expression Omnibus (GEO, https://www.ncbi.nlm. nih.gov/geo/) under accession numbers GSE54388 [6] and GSE74448 [7]. The miRNA expression data in OC were obtained from the GEO under accession numbers GSE83693 [8] and GSE119055 [9]. The expression and prognostic analysis of the mRNAs in OC were obtained from GEPIA and Kaplan-Meier plotter (http://kmplot. com/analysis/index.php). All original data were normalized and $\log 2$-transformed. Differentially expressed genes were identified on the basis of $\mid \log 2$ (fold change) $\mid>1.0$ and $p<0.05$. $\mathrm{R}$ software version 3.4.4 (R Foundation for Statistical Computing, Vienna, Austria) was used for all statistical analyses and plotting.

\section{Identification of $m i R-425-5 p$}

The miRcode is a comprehensive searchable map of putative microRNA target sites in long non-coding RNAs (lncRNAs) [10]. The prediction of target miRNAs of MAGI2-AS3 was carried out through miRcode (http:// www.mircode.org/). Moreover, differentially expressed miRNAs [| log2 (fold change) $\mid>1$ and $\mathrm{p}<0.05$ ] between normal and OC tissues were obtained from the GEO under the accession number GSE83693. Subsequently, the intersections of the predicted miRNAs and upregulated miRNAs in OC were obtained as target miRNAs of
MAGI2-AS3. Finally, the expressions of these miRNAs were further validated using GSE119055.

\section{Integrated target prediction of miR-425-5p}

The target prediction of miRNA was performed through TargetScan (http://www.targetscan.org/), Pictar (https:// pictar.org/), miRanda (http://www.microrna.org/), microT (http://diana.cslab.ece.ntua.gr/microT/), and miRmap (http://mirnamap.mbc.nctu.edu.tw/). In this study, the intersections of the five databases were recognized as the potential target genes of $m i R-425-5 p$. These genes were identified for further analysis.

\section{Correlation analysis}

To analyze the correlations of lncRNA-miRNA, lncRNA-mRNA, and miRNA-mRNA pairs in OC, datasets of IncRNA, miRNA, and mRNA expression in OC samples were downloaded from The Cancer Genome Atlas (TCGA, https://cancergenome.nih.gov/) database. The correlation coefficient was calculated using Spearman's correlation coefficient analysis. The correlation analysis was performed using corrplot $\mathrm{R}$ package, and the visualization was made with ggpubr package in $\mathrm{R}$ software. The $\mathrm{r}>0$ and $p<0.05$ were considered significant positive correlation, whereas $\mathrm{r}<0$ and $p<0.05$ were considered significant negative correlation.

\section{Cell culture}

Human OC cell lines (SKOV-3, A2780, and ES-2) and SV-40 transformed human ovarian epithelial cells (IOSE80) were purchased from the American Type Culture Collection (ATCC, VA, USA). All cells were cultured in Roswell Park Memorial Institute 1640 medium (Gibco; Thermo Fisher Scientific, Inc., Waltham, MA, USA) supplemented with $10 \%$ fetal bovine serum (FBS; Gibco, Thermo Fisher Scientific) and 1\% penicillin/streptomycin (Euroclone S.p.A, Italy) in an incubator at $37^{\circ} \mathrm{C}$ and $5 \%$ $\mathrm{CO}_{2}$.

\section{Cell transfection}

The pcDNA-MAGI2-AS3 vector (MAGI2-AS3), miR425-5p mimics, miR-425-5p inhibitor, pcDNA-AFF4 vector (AFF), and AFF4 shRNAs and their negative control (NC) were obtained from GenePharma, Shanghai, China. pcDNA plasmid without MAGI2-AS3 or AFF4 was used as a NC. The sequences of the miRNA mimic, miRNA inhibitor, AFF4 shRNAs, and their controls are presented in Table S1. Transfection was conducted by using Lipofectamine $^{\mathrm{TM}} 2000$ (Invitrogen; Thermo Fisher Scientific) following the manufacturer's instructions. After transfection, real-time quantitative polymerase chain reaction (RT-qPCR) analysis was performed to assess the transfection efficiency of these genes. 


\section{RT-qPCR analysis}

Total RNA was extracted from cells by using TRIzol reagent (Invitrogen; Thermo Fisher Scientific). cDNA synthesis and RT-qPCR assay for $m i R-425-5 p$ were performed using Hairpin-itTM Real-Time PCR miRNAs kit (GenePharma, Shanghai, China). cDNA syntheses for mRNA and lncRNA were completed using the PrimeScript ${ }^{\mathrm{TM}}$ RT reagent Kit with gDNA Eraser (Takara, Dalian, China), and these cDNA samples were amplified by TB Green ${ }^{\circledR}$ Fast qPCR Mix (Takara) in ABI 7500 Fast Real-Time PCR system (Applied Biosystems, Thermo Fisher Scientific). $\beta$-actin was used as an internal control for mRNAs and lncRNAs, while U6 snRNA was used as an internal control for miRNAs. RT-qPCR was performed in triplicate, and relative gene expression was analyzed by the $2^{-\Delta \Delta \mathrm{Ct}}$ method. The sequences of all primers used in this study are presented in Table S2.

\section{Cell Counting Kit-8 (CCK-8) assay}

The cell viability was assessed by the CCK- 8 assay kit (MilliporeSigma, MA, USA) following the manufacturer's protocol. Briefly, cells were cultured in 96-well plates at a density of $5 \times 104$ cells per well. Consequently, $10 \mu \mathrm{L}$ of the fresh CCK-8 solution was added to each well and then co-incubated for $4 \mathrm{~h}$ at $37^{\circ} \mathrm{C}$. Finally, the optical density of each well was measured at $450 \mathrm{~nm}$ using a microplate reader (Bio-Rad, CA, USA).

\section{Wound-healing assay}

The cell migration capacity was assessed by the woundhealing assay. Briefly, cells were seeded into 6-well plates and incubated for $24 \mathrm{~h}$ to allow attachment. The cell layer was scratched with a $20-\mu \mathrm{L}$ pipette tip, and detached cells were removed carefully by washing with phosphatebuffered saline. The wound area was observed and photographed under optical microscopy (Leica, Germany) after $24 \mathrm{~h}$.

\section{Transwell invasion assay}

The cell invasion capacity was examined using Transwell invasion assay. Briefly, the upper Transwell chambers were pre-coated with Matrigel (BD Biosciences, NJ, USA). The transfected cells were then seeded into the upper chambers that contained a serum-free medium, whereas the lower chambers were filled with medium supplemented with 10\% FBS. Furthermore, the Transwell chambers were incubated for $24 \mathrm{~h}$ at $37^{\circ} \mathrm{C}$. After the incubation, invasive cells were fixed with $4 \%$ paraformaldehyde (Sigma-Aldrich, MO, USA) and then stained with
$0.5 \%$ crystal violet solution. Finally, migratory cells were counted by optical microscopy (Leica, Germany).

\section{Luciferase reporter assays}

To construct the luciferase reporter vector, MAGI2-AS3 and AFF4 3'-untranslated region (3'UTR) wild-type (WT) fragments containing the binding site of miR425-5 $p$ were inserted into the pMir GLO plasmid, while MAGI2-AS3 and AFF4 3'UTR mutant (MUT) fragments were constructed and inserted into the pGL3 pMir GLO plasmid. Briefly, cells were co-transfected with miR-425-5p mimics or miR-NC and WT or MUT vectors using Lipofectamine ${ }^{\mathrm{TM}} 2000$ (Invitrogen; Thermo Fisher Scientific). After transfection for $48 \mathrm{~h}$, cells were fully lysed with passive lysis buffer, and luciferase activity was then detected using a dual-luciferase reporter assay system (Promega, Madison, WI, USA) according to the manufacturer's instructions. The relative luciferase activity was normalized against the Renilla luciferase activity.

\section{Western blotting analysis}

Total protein extracted from cells was placed in radioimmunoprecipitation assay buffer (Vazyme Biotech Co., Ltd., Nanjing, China) supplemented with protease inhibitor cocktail (Roche, Basel, Switzerland), and protein concentrations were measured with the protein $\mathrm{BCA}$ assay kit (Invitrogen; Thermo Fisher Scientific). An equal amount of total protein was separated by $10 \%$ sodium dodecyl sulfate-polyacrylamide gel electrophoresis (Vazyme Biotech Co., Ltd.) and transferred to polyvinylidene fluoride membrane (Invitrogen; Thermo Fisher Scientific). After blocking with 5\% nonfat dried milk, the membrane was incubated with primary antibody against AFF4 (1:1000, Cell Signaling Technology, MA, USA) and $\beta$-action (1:5000, Cell Signaling Technology), followed by horseradish peroxidase-conjugated secondary antibody. Protein detection was visualized with the enhanced chemiluminescent detection reagent (Pierce; Thermo Fisher Scientific) in LI-COR imaging system (LICOR Biosciences, NE, USA). Protein expression levels were quantified by densitometry using Image J software (National Institutes of Health, Bethesda, MD, USA) and normalized to $\beta$-action.

\section{Statistical analysis}

All results were presented as the mean \pm standard error of the mean. Statistical analysis was performed by GraphPad Prism 5.0 (GraphPad Software Inc., CA, USA). Student's t-test was used to compare the difference between the two groups, and one-way analysis of variance test was performed to compare more than two groups; $p<0.05$ was considered significant. 


\section{Results}

MiR-425-5p overexpression in OC was negatively regulated by MAGI2-AS3

We analyzed MAGI2-AS3 expression using GEPIA and GEO datasets (GSE54388 and GSE74448) in OC and normal tissues. Our results showed that MAGI2-AS3 expression was significantly downregulated in $\mathrm{OC}$ tissues compared with that in normal tissues (Fig. S1A). $M A G I 2-A S 3$ was also detected in three OC cell lines (A2780, SKOV3, and ES-2) and IOSE-80 cells and the results demonstrated that MAGI2-AS3 expression was significantly lower in A2780, SKOV3, and ES-2 cells than in IOSE-80 cells (Fig. S1B). Then, miRcode was used to predict the potential binding targets of MAGI2-AS3 and GSE83693 datasets to analyze significantly upregulated miRNAs in OC tissues. Four potential target miRNAs, namely, miR-429, miR-203, miR-210, and miR-425-5p, were obtained by integrating miRcode prediction miRNAs and upregulated miRNAs of GSE83693 datasets (Fig. 1A). Further, GSE119055 datasets were selected to validate the expression of the four miRNAs. The results presented that only $m i R-425-5 p$ displayed significant upregulation in $\mathrm{OC}$ tissues compared with that in ovarian normal tissues (Fig. 1B). The Spearman correlation coefficient analysis revealed that $m i R-425-5 p$ expression was negatively correlated with $M A G I 2-A S 3$ expression in OC (Fig. 1C). Meanwhile, RT-qPCR results demonstrated that $m i R-425-5 p$ was upregulated in OC cell lines (A2780, SKOV3, and ES-2) compared with that in IOSE-80 cells (Fig. 1D). MAGI2-AS3 overexpression could reduce $m i R-$ 425-5p expression in SKOV3 and ES-2 cells (Fig. S1C, Fig. 1E). Furthermore, bioinformatics analyses presented the potential binding sites between $m i R-425-5 p$ and $M A G I-A S 3$ (Fig. 1F). The luciferase reporter assay demonstrated that $m i R-425-5 p$ overexpression significantly suppressed the luciferase activity in WT MAGI2-AS3 3'UTR vector but not in MAGI2-AS3 3'UTR MUT vector in SKOV3 and ES-2 cells (Fig. 1G), indicating the direct interactions between $m i R-425-5 p$ and MAGI2$A S 3$. Taken together, our data supported the finding that an abnormally high level of $m i R-425-5 p$ expression in $\mathrm{OC}$ was directly negatively regulated by MAGI2-AS3.

\section{Silencing miR-425-5p suppressed the proliferation, migration, and invasion of $\mathrm{OC}$ cells}

To further investigate the role of $m i R-425-5 p$ in OC progression, the $m i R-425-5 p$ inhibitor or NC inhibitor was transfected into OC cells, namely, SKOV3 and ES-2 by using Lipofectamine ${ }^{\mathrm{TM}} 2000$, respectively. The suppression efficiency of the miR-425-5p inhibitor after transfection into SKOV3 and ES-2 cells was confirmed by RT-qPCR, and the results indicated that $m i R-425-5 p$ expression was significantly downregulated in OC cells (SKOV3 and ES-2) treated with the miR-425-5p inhibitor compared with that in OC cells (SKOV3 and ES-2) treated with the $\mathrm{NC}$ inhibitor (Fig. S2). Then, the results of the CCK-8 assays indicated that silencing $m i R-425-5 p$ markedly suppressed the cell proliferation capacity of SKOV3 and ES-2 cells (Fig. 2A). The results of the wound-healing assay suggested that silencing $m i R-425-5 p$ significantly inhibited the migration of SKOV3 and ES-2 cells (Fig. 2B). Meanwhile, the results of the Transwell invasion assay indicated that silencing $m i R-425-5 p$ significantly decreased the invasive abilities of SKOV3 and ES-2 cells (Fig. 2C). These results indicated that silencing $m i R-425-5 p$ could inhibit the malignant phenotype of OC cells, thus confirming the oncogenic role of $m i R-425-5 p$ in OC cells.

\section{$A F F 4$, as a new target of $m i R-425-5 p$, inhibited the proliferation, migration, and invasion of OC cells}

To understand further the molecular mechanisms of $m i R-425-5 p$ in OC, we predicted the potential targets of $m i R-425-5 p$ using five recognizable and promising miRNA target prediction tools, namely, TargetScan, PicTar, miRanda, microT, and miRmap. The results demonstrated that 29 mRNAs are shared in these five tools (Fig. 3A). Further, to understand the roles of these 29 genes in $\mathrm{OC}$, we used GEPIA to analyze the expression patterns of these genes in $\mathrm{OC}$ and normal tissues. The results indicated that only 10 of 29 genes were significantly dysregulated in $\mathrm{OC}$ samples compared with that in normal ovarian samples (Fig. S3A). Subsequently, the correlations between these 10 dysregulated genes and $m i R-425-5 p$ were analyzed using the TCGA database of OC. The results indicated that $m i R-425-5 p$ was significantly negatively correlated with only 4 of 10 dysregulated genes, including $A F F 4, M E F 2 C, P R D M 8$, and DIP2C (Fig. S3B). Based on the miRNA sponge hypothesis, these four genes might be promising targets of $m i R$ $425-5 p$ (Fig. S3C). Further, the prognostic values of these four genes in $\mathrm{OC}$ were determined by Kaplan-Meier plotter, and the results exposed that the expression levels of $M E F 2 C$ and DIP2C were not associated with the overall survival of patients with $\mathrm{OC}$, and the high PRDM8 expression was negatively correlated with the overall survival of patients with OC, while AFF4 exhibited the opposite correlation: the high AFF4 expression indicated a better prognosis (Fig. S4). The comprehensive analysis of the above expression analysis, survival analysis, and correlation analysis suggested that AFF4 played a vital role in the pathogenesis of $\mathrm{OC}$ and might be the most promising target gene of $m i R-425-5 p$.

To determine whether AFF4 was the target gene of $m i R-425-5 p$ in $\mathrm{OC}$, the mRNA expression of $A F F 4$ was examined in several OC cell lines (A2780, SKOV3, 

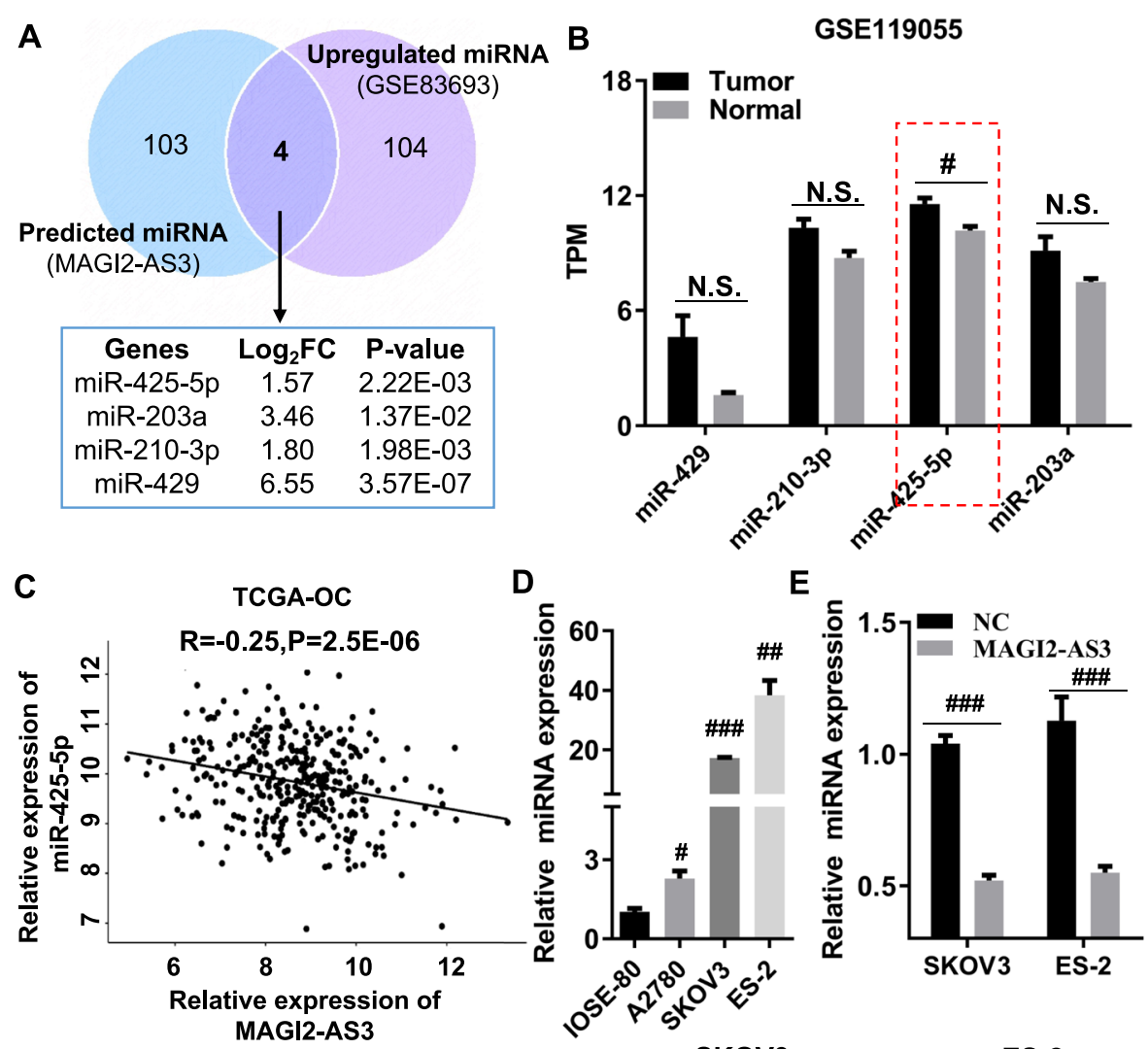

$\mathbf{F}$

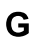

SKOV3

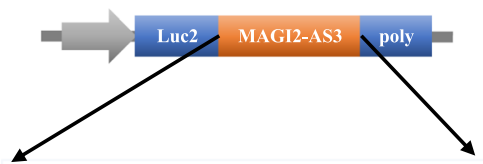

WT MAGI2-AS3:5' tactgtagcattcacGTGTCtct $3^{\prime}$ miR-425-5p: 3' || aguug || MUT MAGI2-AS3:5' tactgtagcattcacCACAGtct 3
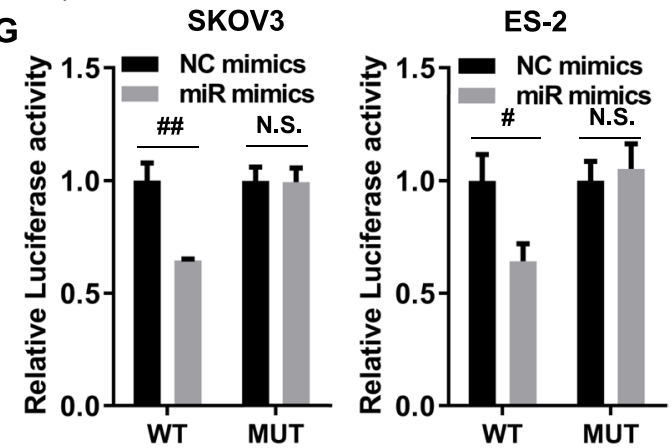

Fig. 1 miR-425-5p overexpression in ovarian cancer is negatively regulated by MAG/2-AS3. A Venn diagram showing the overlapping of the predicted target miRNAs and upregulated miRNAs in GSE83693. GSE83693 contained four normal ovarian tissue samples and 16 ovarian cancer samples. B GSE119055 datasets were used to verify the expression levels of four miRNAs in ovarian cancer tissues and normal ovarian tissues. GSE1 19055 contained three normal ovarian tissue samples and six ovarian cancer samples. C Correlation of MAG/2-AS3 and miR-425-5p expression. DmiR-425-5p expression was detected by real-time quantitative polymerase chain reaction (RT-qPCR) in ovarian cancer cells (A2780, SKOV3, and ES-2) and SV-40 transformed human ovarian epithelial cells (IOSE-80) cells. EMiR-425-5p expression levels were determined by RT-qPCR in SKOV-3 and ES-2 cells, after transient transfection of MAGI2-AS3 and its negative control (NC). F Schematic diagram showing the binding sites of miR-425-5p and MAGI2-AS3 3'UTR and the mutation site MAG/2-AS3 3'UTR. G Relative luciferase activities were detected by dual-luciferase reporter assays in SKOV-3 and ES-2 cells after co-transfection of miR-425-5p mimics or NC mimics with wild-type-MAGI2-AS3-luc (WT) and mutant-MAGI2-AS3-IuC (MUT). ${ }^{N . S} \cdot p>0.05,{ }^{\#} p<0.05,{ }^{\# \#} p<0.01,{ }^{\# \#} p<0.001$

and ES-2) and IOSE-80 cells by RT-qPCR. The results revealed that the mRNA expression of AFF4 was significantly downregulated in A2780, SKOV3, and ES-2 cells compared with that in IOSE-80 cells (Fig. 3B). Bioinformatics analysis suggested the potential binding sites of miR-425-5p in AFF4 $3^{\prime}$-UTR (Fig. 3C). To further validate whether $m i R-425-5 p$ directly targets the AFF4 3'-UTR by a dual-luciferase reporter gene assay, the WT or MUT AFF4 3'-UTR dual-luciferase plasmids and miR-425-5p mimics or NC mimics were cotransfected into OC cells. As a result, the miR-425-5p overexpression significantly suppressed the luciferase 

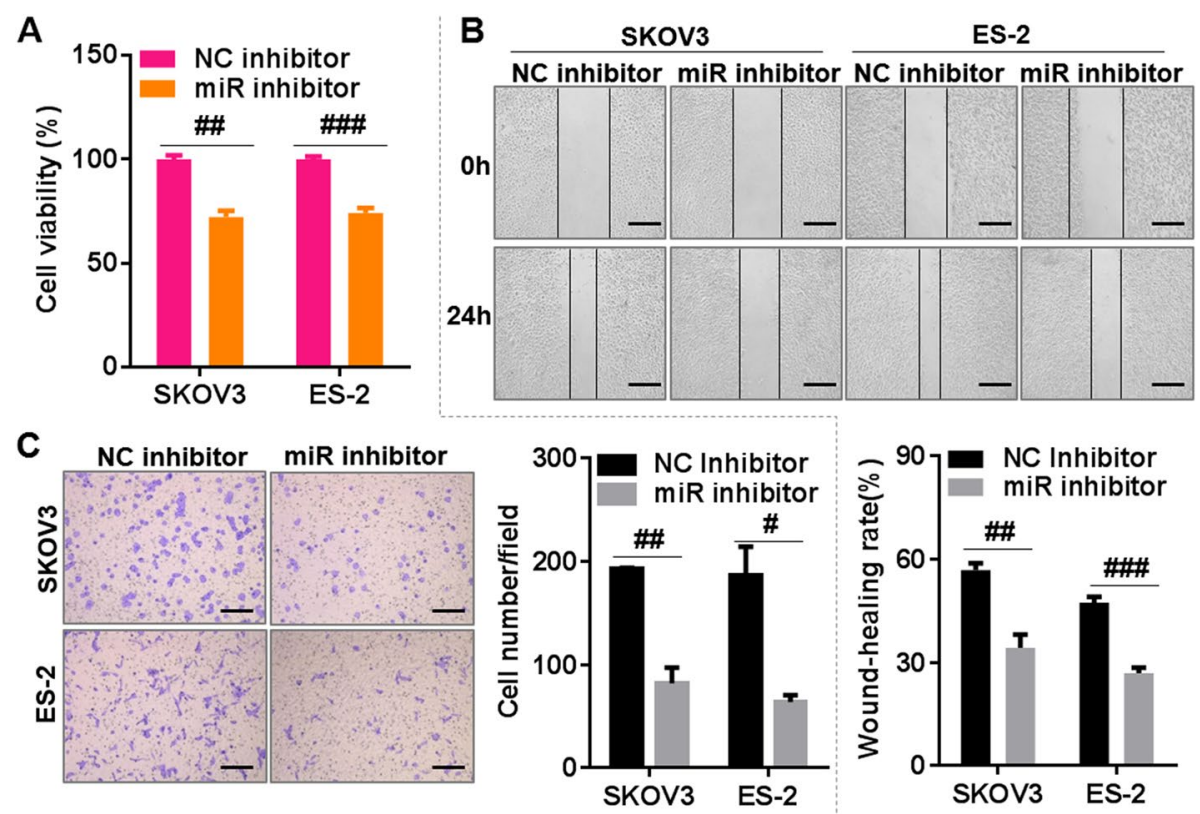

Fig. 2 Knockdown of miR-425-5p inhibits the proliferation, migration, and invasion of ovarian cancer cells. A Cell proliferation capacity was measured using Cell Counting Kit-8 assays in miR-425-5p inhibitor (miR inhibitor), or its negative control inhibitor (NC inhibitor), in transfected SKOV-3 and ES-2 cells. B Cell migration capacity was measured using wound-healing assays in miR-425-5p inhibitor (miR inhibitor), or its NC inhibitor, in transfected SKOV-3 and ES-2 cells. C Cell invasion capacity was measured using Transwell assays in miR-425-5p inhibitor (miR inhibitor), or its NC inhibitor, in transfected SKOV-3 and ES-2 cells. Bar $=100 \mu \mathrm{m} .{ }^{\#} p<0.05,{ }^{\# \#} p<0.01,{ }^{\# \# \#} p<0.001$

activity of the WT AFF4 3'UTR reporter vector but not the MUT AFF4 3'UTR reporter vector in SKOV-3 and ES-2 cells (Fig. 3D). In addition, silencing of the miR425-5p expression significantly upregulated the mRNA and protein expression of AFF4 in SKOV3 and ES-2 cells (Fig. 3E, F), suggesting that miR-425-5p negatively regulated AFF4 expression in SKOV3 and ES-2 cells. Altogether, our results indicated that $m i R-425-5 p$ binds directly to the 3'UTR of AFF4 to inhibit its expression in OC cells.

To further investigate the function of AFF4 in OC, SKOV-3 and ES-2 cells were transfected with overexpression $A F F 4$ vector ( $A F F 4$ ) or its NC vector. The results presented that the mRNA expression of AFF4 significantly was upregulated in SKOV-3 and ES-2 cells transfected with AFF4 compared with that in NC-treated SKOV-3 and ES-2 cells (Fig. 4A). The results of the CCK-8 assays indicated that AFF4 overexpression suppressed the proliferation of SKOV-3 and ES-2 cells (Fig. 4B). The results of the wound-healing assay indicated that AFF4 overexpression significantly inhibited the migration ability of SKOV-3 and ES-2 cells (Fig. 4C, D). Meanwhile, the results of the Transwell invasion assay indicated that AFF4 overexpression significantly reduced the invasive ability of SKOV-3 and ES-2 cells (Fig. 4E, F). Altogether, these data indicated that AFF4 overexpression could inhibit the malignant phenotype of $\mathrm{OC}$ cells, suggesting the antioncogenic role of AFF4 in OC cells.

\section{Silencing miR-425-5p suppressed the malignant phenotype of $\mathrm{OC}$ cells by upregulating AFF4 expression}

To further explore whether $m i R-425-5 p$ inhibited malignant phenotypes in OC cells by regulating AFF4 expression, we transfected the constructed AFF4 shRNAs (sh-AFF4\#1, sh-AFF4\#2, and sh-AFF4\#3) and their NC (sh-NC) into OC cells and selected the sh-AFF4 with the best inhibitory effect for the subsequent experiments. The results of the RT-qPCR analysis revealed that all AFF4 shRNAs (sh-AFF4\#1, sh-AFF4\#2, and sh-AFF4\#3) significantly inhibited AFF4 expression in SKOV-3 and ES-2 cells, and sh-AFF4\#1 (sh-AFF4) was the most effective and could be used for subsequent studies (Fig. S5A). Furthermore, the miR-425-5p inhibitor or sh-AFF4 was transfected into OC cells separately or co-transfected with Lipofectamine ${ }^{\mathrm{TM}} 2000$ reagent, and the promotion effect of silencing miR-425-5p expression on the mRNA and protein expression of AFF4 in SKOV-3 and ES-2 cells was significantly inhibited by the transfection of $A F F 4$ shRNA (Fig. S5B, C). The results of the CCK-8 assay indicated that silencing AFF4 eradicated the inhibitory effect of silencing $m i R-425-5 p$ on the cell proliferation capacity of SKOV-3 and ES-2 cells (Fig. 5A). The results 

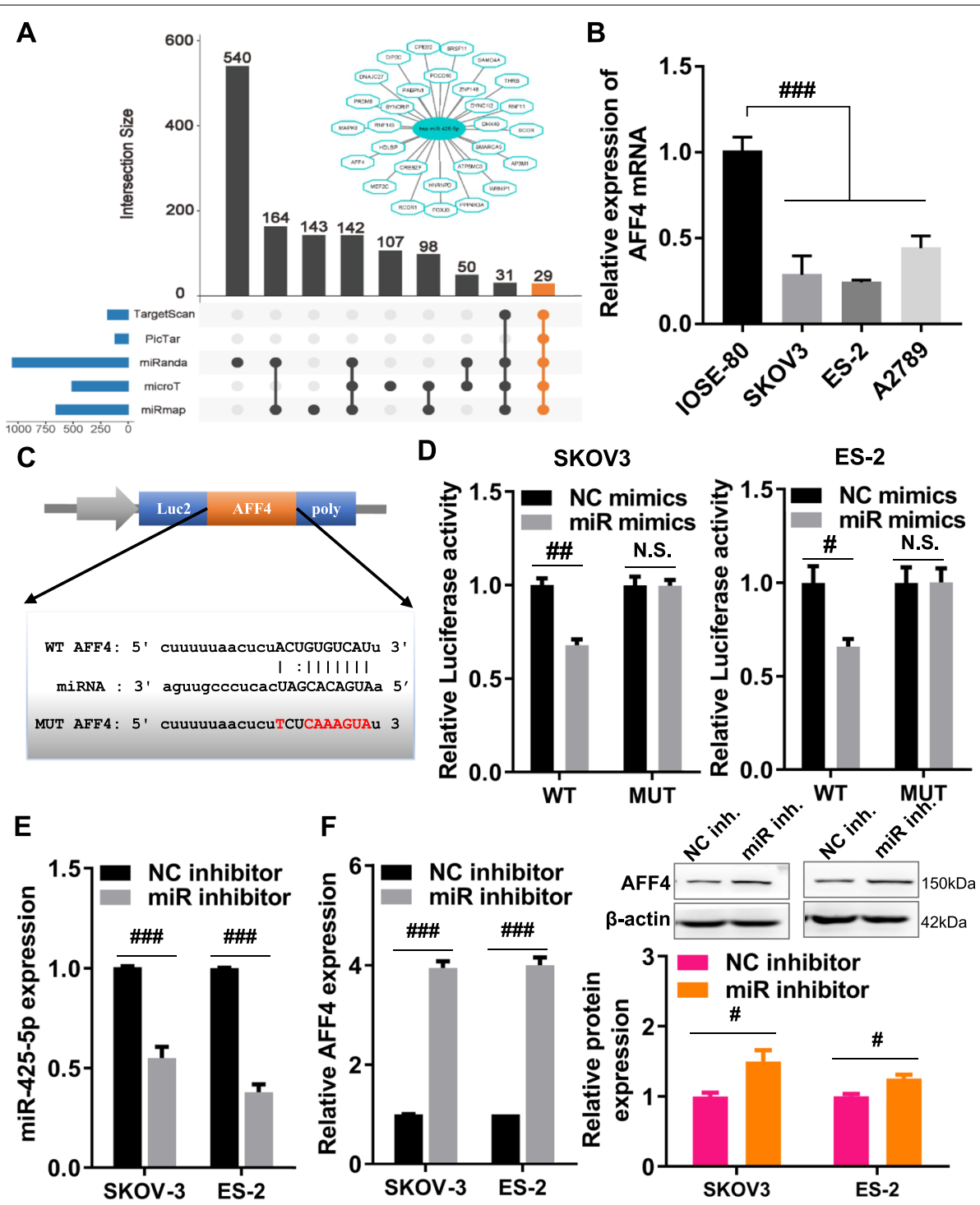

Fig. 3 AFF4 is a direct target of miR-425-5p in ovarian cancer cells. A To predict potential targets of miR-425-5p using multiple miRNA-target prediction tools, the miR-425-5p-target interaction network was visualized using Cytoscape 3.7.1. BAFF4 expression in ovarian cancer cells (A2780, SKOV3, and ES-2) and IOSE-80 cells was detected by real-time quantitative polymerase chain reaction (RT-qPCR). C Schematic diagram showing the binding sites of miR-425-5p and AFF4 3'UTR and the mutation site of AFF4 3'UTR. D Relative luciferase activities were detected by dual-luciferase reporter assays in SKOV-3 and ES-2 cells after co-transfection of miR-425-5p mimics (miR mimics) or negative control mimics (NC mimics) with wild-type-AFF4-Iuc (WT) and mutant-AFF4-luc (MUT). EMiR-425-5p expression in SKOV-3 and ES-2 cells was determined by RT-qPCR, after transient transfection of miR-425-5p inhibitor (miR inhibitor) and negative control inhibitor (NC inhibitor). F The mRNA and protein expressions of AFF4 in SKOV-3 and ES-2 cells were determined by RT-qPCR and western blot analysis, after transient transfection of miR-425-5p inhibitor (miR inhibitor) and $N C$ inhibitor. miR inhibitor, miR-425-5p inhibitor; NC inhibitor, corresponding negative control of the miR-425-5p inhibitor; miR mimics, miR-425-5p mimics; NC mimics, negative control of miR-425-5p mimics. N.S. $p>0.05, " \#<0.05,{ }^{\# \#} p<0.01,{ }^{\# \# \#} p<0.001$

of the wound-healing assay indicated that silencing AFF4 dramatically weakened the inhibitory effect of silencing $m i R-425-5 p$ on the cell migration capacity of SKOV-3 and ES-2 cells (Fig. 5B, C). Meanwhile, the results of the Transwell invasion assay indicated that silencing AFF4 dramatically attenuated the inhibitory effect of silencing $m i R-425-5 p$ on the cell invasion capacity of SKOV-3 and ES-2 cells (Fig. 5D, E). Moreover, silencing AFF4 could promote the proliferation, migration, and invasion of SKOV-3 and ES-2 cells (Fig. 5A-E), suggesting that silencing AFF4 promoted the malignant phenotype of OC cells. Taken together, these results suggested that silencing miR-425-5p inhibited the malignant phenotype of OC cells by upregulating AFF4 expression. 

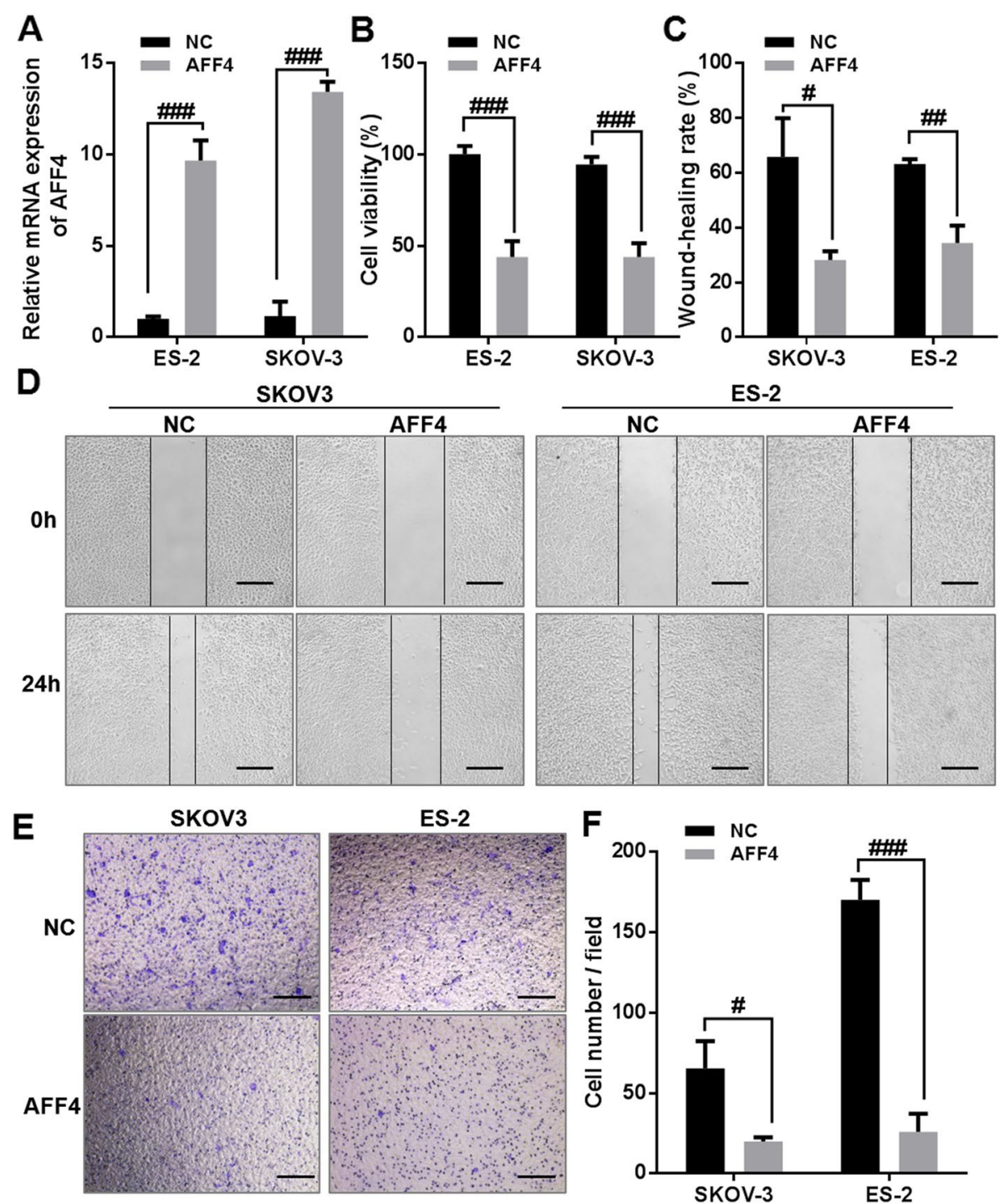

Fig. 4 AFF4 overexpression inhibits the proliferation, migration, and invasion of ovarian cancer cells. A AFF4 mRNA expression was determined by a real-time quantitative polymerase chain reaction in SKOV-3 and ES-2 cells, after transient transfection of the overexpression AFF4 vector (AFF4) or its negative control vector (NC). B Cell proliferation capacity was measured using Cell Counting Kit-8 assays in the overexpression AFF4 vector (AFF4), or its negative control vector (NC), in transfected SKOV-3 and ES-2 cells. C, D Cell migration capacity was measured using wound-healing assays in the overexpression AFF4 vector (AFF4), or its negative control (NC) vector, in transfected SKOV-3 and ES-2 cells. E, F Cell invasion capacity was measured using Transwell assays in the overexpression AFF4 vector (AFF4), or its NC vector, in transfected SKOV-3 and ES-2 cells. Bar $=100 \mu \mathrm{m}$. ${ }^{\#} p<0.05, \# p<0.01, \# \# p<0.001$

\section{Discussion}

$\mathrm{OC}$ is one of the most common cancers among women with the third highest incidence rate and the highest mortality rate worldwide [2]. This is due to the insidious onset of $\mathrm{OC}$ and the lack of specific early diagnosis and effective long-term therapies. Recent studies are focusing on the development of new diagnostic and therapeutic biomarkers to improve the survival rates [11]. To the best of our knowledge, this was the first study to identify that miR425-5 $p$ overexpression in $\mathrm{OC}$ was negatively regulated by
MAGI2-AS3 and that silencing $m i R-425-5 p$ inhibited the proliferation, migration, and invasion of OC cells by targeting AFF4, suggesting that miR-425-5p/AFF4 signaling may be a novel therapeutic target for patients with $\mathrm{OC}$.

$M A G I 2-A S 3$ plays a critical role in various cancers [12]. In this study, we demonstrated that MAGI2-AS3 is significantly downregulated in $\mathrm{OC}$ tissues and cell lines, consistent with the results of a previous study [13]. MAGI2-AS3 is considered an important player in high-grade serous ovarian carcinoma, regulating the 

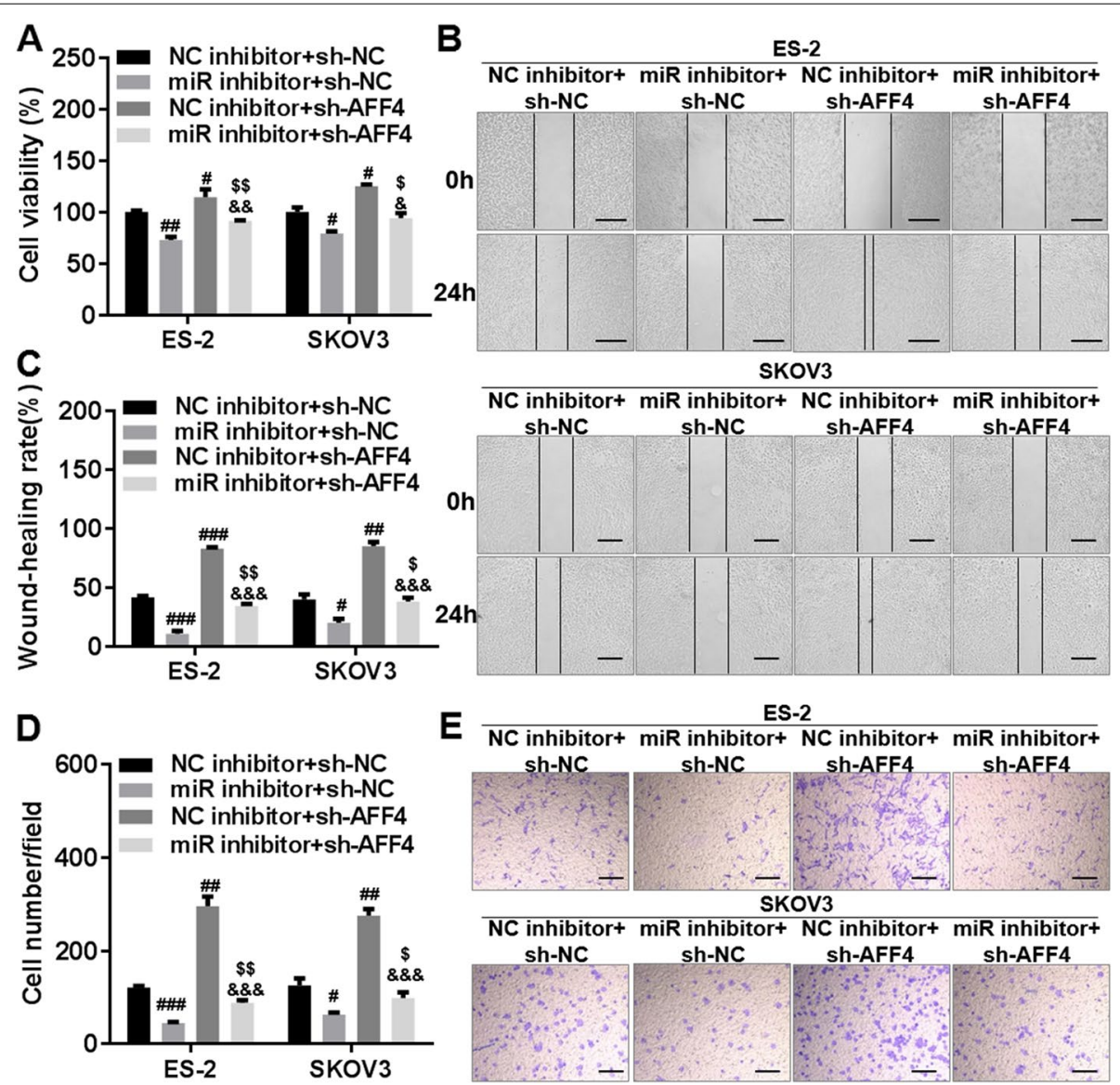

Fig. 5 Silencing AAF4 against miR-425-5p affects the proliferation, invasion, and migration of ovarian cancer cells. A The Cell Counting Kit-8 assay was used to detect cell proliferation in transfected SKOV-3 and ES-2 cells. B, C The wound-healing assay was used to detect cell migrations in transfected SKOV-3 and ES-2 cells. D, E The Transwell assay was used to detect cell invasions in transfected SKOV-3 and ES-2 cells. Bar $=100 \mu \mathrm{m}$. sh-AFF4, AFF4 small hairpin RNA; sh-NC, negative control of sh-AFF4; miR inhibitor, miR-425-5p inhibitor; NC inhibitor, negative control of miR-425-5p inhibitor. ${ }^{\#} p<0.05,{ }^{\# \#} p<0.001,{ }^{\$} p<0.05$, and ${ }^{\$ \$} p<0.01$ comparison with miR inhibitor + sh-NC. ${ }^{\&} p<0.05$, \&\& $p<0.01$, and ${ }^{\text {\&\& }} p<0.001$ comparison with NC inhibitor + sh-AFF4

downstream mRNA expression by sponging miR-15-5p, miR-374a-5p, and miR-374b-5p in the competing endogenous RNA (ceRNA) regulation network [13]. This study have suggested the suppressor role of MAGI2-AS3 in OC progression. In addition, lncRNAs may act as competing endogenous RNA (ceRNAs) by repressing the expression of miRNAs and further regulating the associated mRNAs [14]. LINC00511 mediates the effects of ESR1 on proliferation and invasion of OC through miR-424-5p and $m i R-370-5 p$ [15]. LncRNA PTAR promotes epithelial-mesenchymal transition (EMT) and invasion-metastasis in serous OC by competitively binding miR-101-3p to regulate $Z E B 1$ expression [16]. Based on a comprehensive strategy of data mining, computational biology, and RT-PCR, we identified that miR-425-5p was significantly upregulated in OC and may be involved in OC development. Recent studies have reported that miR-425-5p may be involved in OC progression, but its specific role and regulatory mechanism are unclear [17]. Our results indicated that silencing miR-425-5p inhibited the proliferation, migration, and invasion of OC cells, which was consistent with its role in other cancers. For example, miR-425-5p is significantly increased in hepatocellular carcinoma (HCC) tissues and is closely related to the poor prognosis of patients with HCC, which as an oncogene promotes the proliferation and migration of HCC cells via $R N F 11$ or FOXD3 $[18,19]$. In colorectal cancer, miR$425-5 p$ promotes cell proliferation, migration, invasion, and EMT by activating the CTNND1-mediated $\beta$-catenin pathway [20]. In breast cancer, $m i R-425-5 p$ promotes cell growth by target binding to the PTEN 3'UTR [21]. In lung cancer, $m i R-425-5 p$ overexpression is positively associated with poor prognosis and enhances the proliferation, migration, and invasion of cells [22, 23]. In renal cell carcinoma, studies have shown that $m i R-425-5 p$ is an oncogene in this cancer [24]. The aforementioned studies have 
suggested that $m i R-425-5 p$ may act as an oncogene in many cancers, and targeting $m i R-425-5 p$ may be a potential method for the treatment of cancer in the future.

AFF4 (AF4/FMR2), as a core component of the super elongation complex [25], is involved in the progression of cancer, e.g., leukemia [26], head and neck squamous cell carcinoma [27], melanoma [28], bladder cancer [29, 30], and lung cancer [31]. However, the role of AFF4 in OC development remains unknown. To the best of our knowledge, we are the first to find that AFF4 was significantly decreased in OC tissues and cells and was closely related to the good prognosis of patients with OC. Further, our results indicated that AFF4 overexpression inhibited the proliferation, migration, and invasion of OC cells in vitro. By contrast, silencing AFF4 promoted the proliferation, migration, and invasion of OC cells in vitro, suggesting that $A F F 4$ played a tumorsuppressive role in OC. However, AFF4 played a major oncogenic role in several cancers [26-31], in contrast to its role in OC. A possible reason is that AFF4, as a transcription factor, mainly activated tumor-suppressor genes or signaling pathways in OC; thus, the specific regulatory mechanism needs to be further explored. Finally, our data demonstrated that AFF4 was the new target of $m i R-425-5 p$, and silencing AFF4 reversed the malignant phenotype-suppressing effects of miR-425-5p knockdown in SKOV-3 and ES-2 cells. Herein, we first revealed that miR-425-5p demonstrated oncogenic actions by directly downregulating AFF4 in OC.

However, this study has two main limitations. First, although silencing miR-425-5p can inhibit malignant phenotypes in OC cells, we have not verified whether $m i R-425-5 p$ overexpression promotes malignant phenotypes in OC cells. Second, the role of the miR-425-5p/ AFF4 pathway in OC progression has not been further verified in vivo. Thus, the downstream signaling of $A F F 4$ in $\mathrm{OC}$ remains largely unknown. In the further study, these parts in our experiment will be designed.

\section{Conclusions}

The results of this study showed, for the first time, that $m i R-425-5 p$ was significantly upregulated in OC tissues and cells, and it was negatively regulated by MAGI2-AS3. Further, $A F F 4$, which acts as a tumor-suppressor gene in $\mathrm{OC}$, was the new target for miR-425-5p. Finally, silencing $m i R-425-5 p$ suppressed the proliferation, migration, and invasion of OC cells by targeting upregulated AFF4. Our findings suggested that miR-425-5p/AFF4 signaling may be a potential therapeutic target for patients with $\mathrm{OC}$ in the future.

\section{Abbreviations}

OC: Ovarian cancer; miRNAs: MicroRNAs; AFF4: AF4/FMR2 family member 4; GEPIA: Gene Expression Profiling Interactive Analysis; GEO: Gene Expression Omnibus; LncRNAs: Long non-coding RNAs; TCGA: The Cancer Genome Atlas; RT-qPCR: Real-time quantitative polymerase chain reaction; CCK-8: Cell Counting Kit-8; 3'UTR: 3'-untranslated region; ceRNA: Competing endogenous RNA; HCC: Hepatocellular carcinoma.

\section{Supplementary Information}

The online version contains supplementary material available at https://doi. org/10.1186/s13048-021-00894-x.

\begin{abstract}
Additional file 1: Figure S1. MAG/2-AS3 expression in ovarian cancer tissues and cells. (A) MAGI2-AS3 was downregulated in ovarian cancer tissues based on Gene Expression Profiling Interactive Analysis and Gene Expression Omnibus. GSE54388 contained six normal ovarian tissue samples and 12 ovarian cancer samples. GSE74448 contained 11 normal ovarian tissue samples and 29 ovarian cancer samples. (B) The level of MAG/2-AS3 expression in IOSE-80, A2780, SKOV3, and ES-2 cells was detected by real-time quantitative polymerase chain reaction (RT-qPCR). (C) The levels of MAG12-AS3 expression in SKOV-3 and ES-2 cells were determined by RT-qPCR, after transient transfection of MAG/2-AS3 and its negative control. \# $p<0.05, \# p<0.01, \# \#<0.001$
\end{abstract}

Additional file 2: Figure S2. MiR-425-5p expression levels were determined by real-time quantitative polymerase chain reaction in SKOV-3 and ES-2 cells, after transient transfection of miR-425-5p mimics and negative control mimics. ${ }^{\#} p<0.05,{ }^{\# \# \#} p<0.001$.

Additional file 3: Figure S3. Identification of potential target genes of miR-425-5p. (A) The expressions of 29 target genes in ovarian cancer tissues were analyzed by GEPIA data of ovarian cancer (only showed different target genes). (B) Correlations between miR-425-5p and different target genes in ovarian cancer (only showed significant correlation). (C) Overview of genes negatively associated with miR-425-5p.

Additional file 4: Figure S4. Overall survival rate of four hub targets by Kaplan-Meier survival analysis in ovarian cancer.

Additional file 5: Figure S5. Effects of AFF4 knockdown on miR-425-5p regulation. (A) The levels of AFF4 expression were determined by real-time quantitative polymerase chain reaction (RT-qPCR) in SKOV-3 and ES-2 cells, after transient transfection of AFF4 shRNA\#1 (sh-AFF-1), AFF4 shRNA\#2 (sh-AFF-2), AFF4 shRNA\#3 (sh-AFF-3), and its negative control (sh-NC). (B) The mRNA expression of AFF4 was determined by RT-qPCR in SKOV-3 and ES-2 cells with different treatments. (C) The protein expression of AFF4 was determined by western blot analyses in SKOV-3 and ES-2 cells with different treatments. sh-AFF4, AFF4 small hairpin RNA; sh-NC, negative control of sh-AFF4; miR inhibitor, miR-425-5p inhibitor; NC inhibitor, negative control of miR-425-5p inhibitor. ${ }^{\#} p<0.05$, ${ }^{\# \#} p<0.001 .{ }^{\$} p<0.05,{ }^{\$} p<0.01$ comparison with miR inhibitor + sh-NC. ${ }^{\&} p<0.05$ comparison with NC inhibitor + sh-AFF4.

Additional file 6: Table S1. Sequences of miR-425-5p mimic, miR-425-5p inhibitor, and sh-AFF4 that were used in the study.

Additional file 7: Table S2. Primer sequences of MAGI2-AS3, miR-425-5p, AFF4, U6 and GAPDH.

\section{Acknowledgments}

We would like to thank the open-access public dataset from the GEPIA, TCGA, Kaplan-Meier Plotter and GEO databases.

\section{Authors' contributions}

The author ZHW contributed to the data collection and finished the draft of the manuscript. YZ and HPM performed the data analysis. JLG performed the drawing and revision of all figure and tables. YRT participated in the coordination of later research, as well as the revision and final review the manuscript, and was a major contributor in writing the manuscript. JHL and HPM provided the laboratory for the authors. All the authors read and approved the final manuscript. 


\section{Funding}

Not applicable.

\section{Availability of data and materials}

The datasets used and/or analysed during the current study are available from the corresponding author on reasonable request.

\section{Declarations}

Ethics approval and consent to participate Not applicable.

\section{Consent for publication}

Not applicable.

\section{Competing interests}

The authors declare that they have no competing interests.

\section{Author details}

${ }^{1}$ Department of Clinical Laboratory, Fujian Provincial Maternity and Children Hospital, Affiliated Hospital of Fujian Medical University, Fuzhou 350001, Fujian Province, China. ${ }^{2}$ Department of Laboratory, Second People's Hospital, Kashgar Area, Xinjiang 844000, China. ${ }^{3}$ Department of Clinical Laboratory, Tuoli County People's Hospital, Tacheng, Xinjiang 834500, Uygur Autonomous Region, China. ${ }^{4}$ Department of Clinical Laboratory, Beijing Rehabilitation Hospital, Capital Medical University, Beijing 100144, China. ${ }^{5}$ Department of Clinical Laboratory, Children's Hospital of Xinjiang Uygur Autonomous Region, Urumqi, Xinjiang 830054, Uygur Autonomous Region, China. ' ${ }^{6}$ Laboratory Department of Shengli Oilfield Central Hospital, Dongying 257100, China.

\section{Received: 13 July 2021 Accepted: 11 October 2021}

Published online: 22 October 2021

\section{References}

1. Overman VP. American Cancer Society. Int J Dent Hyg. 2006;4(2):109.

2. Corney DC, Nikitin AY. MicroRNA and ovarian cancer. Histol Histopathol. 2008;23:1161-9.

3. Peng Y, Croce CM. The role of MicroRNAs in human cancer. Signal Transduct Target Ther. 2016;1:15004.

4. Sun $L$, Jiang $R$, $L i$ J, Wang $B, M a C$, Lv Y, et al. MicoRNA-425-5p is a potential prognostic biomarker for cervical cancer. Ann Clin Biochem. 2016;54:127-33.

5. Yang $G$, Zhang C, Wang N, Chen J. miR-425-5p decreases LncRNA MALAT1 and TUG1 expressions and suppresses tumorigenesis in osteosarcoma via Wnt/ $\beta$-catenin signaling pathway. Int J Biochem Cell Biol. 2019;111:42-51.

6. Yeung TL, Leung CS, Wong KK, Gutierrez-Hartmann A, Kwong J, Gershenson DM, et al. ELF3 is a negative regulator of epithelial-mesenchymal transition in ovarian cancer cells. Oncotarget. 2017;8:16951-63.

7. Tang Z, Li C, Kang B, Gao G, Li C, Zhang Z. GEPIA: a web server for cancer and normal gene expression profiling and interactive analyses. Nucleic Acids Res. 2017;45:W98-W102.

8. Nam EJ, Kim S, Lee TS, Kim HJ, Lee JY, Kim SW, et al. Primary and recurrent ovarian high-grade serous carcinomas display similar microRNA expression patterns relative to those of normal ovarian tissue. Oncotarget. 2016;7:70524-34.

9. Dong $S$, Wang $R$, Wang H, Ding Q, Zhou X, Wang J, et al. HOXD-AS1 promotes the epithelial to mesenchymal transition of ovarian cancer cells by regulating miR-186-5p and PIK3R3. J Exp Clin Cancer Res. 2019;38:110.

10. Jeggari A, Marks DS, Larsson E. miRcode: a map of putative microRNA target sites in the long non-coding transcriptome. Bioinformatics (Oxford, England). 2012;28:2062-3.

11. Arend RC, Londono-Joshi Al, Straughn JM Jr, Buchsbaum DJ. The Wnt/ beta-catenin pathway in ovarian cancer: a review. Gynecol Oncol. 2013;131:772-9.
12. Liu Q, Liu S, Wang X, Zhang J, Liu K. LncRNA MAGI2-AS3 is involved in cervical squamous cell carcinoma development through CDK6 upregulation. Infect Agent Cancer. 2019;14:37.

13. Ren H, Li Z, Tang Z, Li J, Lang X. Long noncoding MAGI2-AS3 promotes colorectal cancer progression through regulating miR-3163/TMEM106B axis. J Cell Physiol. 2020;235:4824-33.

14. Fang Y, Fullwood MJ. Roles, functions, and mechanisms of long non-coding RNAs in cancer. Genomics Proteomics Bioinformatics. 2016;14:42-54.

15. Wang K, Zhu G, Bao S, Chen S. Long non-coding RNA LINC00511 mediates the effects of ESR1 on proliferation and invasion of ovarian cancer through miR-424-5p and miR-370-5p. Cancer Manag Res. 2019;11:10807-19.

16. Liang $H, Y U T$, Han $Y$, Jiang $H$, Wang $C$, You T, et al. LncRNA PTAR promotes EMT and invasion-metastasis in serous ovarian cancer by competitively binding miR-101-3p to regulate ZEB1 expression. Mol Cancer. 2018;17:119.

17. Gao L, Li X, Nie X, Guo Q, Liu Q, Qi Y, et al. Construction of novel mRNAmiRNA-IncRNA regulatory networks associated with prognosis of ovarian cancer. J Cancer. 2020;11:7057-72.

18. Rao D, Guan S, Huang J, Chang Q, Duan S. miR-425-5p acts as a molecular marker and promoted proliferation, migration by targeting RNF11 in hepatocellular carcinoma. Biomed Res Int. 2020;2020:6530973.

19. Wu H, Shang J, Zhan W, Liu J, Ning H, Chen N. miR-425-5p promotes cell proliferation, migration and invasion by directly targeting FOXD3 in hepatocellular carcinoma cells. Mol Med Rep. 2019;20:1883-92.

20. Liu D, Zhang H, Cui M, Chen C, Feng Y. Hsa-miR-425-5p promotes tumor growth and metastasis by activating the CTNND1-mediated $\beta$-catenin pathway and EMT in colorectal cancer. Cell Cycle (Georgetown, Tex). 2020;19:1917-27.

21. Zhang LF, Zhang JG, Zhou H, Dai TT, Guo FB, Xu SY, et al. MicroRNA425-5p promotes breast cancer cell growth by inducing PI3K/AKT signaling. Kaohsiung J Med Sci. 2020;36:250-6.

22. FuY, LiY, Wang $X, L i F$, Lu Y. Overexpression of miR-425-5p is associated with poor prognosis and tumor progression in non-small cell lung cancer. Cancer Biomark. 2020;27:147-56.

23. Zhou JS, Yang ZS, Cheng SY, Yu JH, Huang CJ, Feng Q. miRNA-425-5p enhances lung cancer growth via the PTEN/PI3K/AKT signaling axis. BMC Pulm Med. 2020;20:223.

24. Quan J, Li Y, Pan X, Lai Y, He T, Lin C, et al. Oncogenic miR-425-5p is associated with cellular migration, proliferation and apoptosis in renal cell carcinoma. Oncol Lett. 2018;16:2175-84.

25. Zhou C-C, Xiong Q-C, Zhu X-X, Du W, Deng P, Li X-B, et al. AFF1 and AFF4 differentially regulate the osteogenic differentiation of human MSCS. Bone Res. 2017;5:17044.

26. Komori T, Doi A, Nosaka T, Furuta H, Akamizu T, Kitamura T, et al. Regulation of AMP-activated protein kinase signaling by AFF4 protein, member of AF4 (ALL1-fused gene from chromosome 4) family of transcription factors, in hypothalamic neurons. J Biol Chem. 2012;287:19985-96.

27. Deng P, Wang J, Zhang X, Wu X, Ji N, Li J, et al. AFF4 promotes tumorigenesis and tumor-initiation capacity of head and neck squamous cell carcinoma cells by regulating SOX2. Carcinogenesis. 2018;39:937-47.

28. Hu H, Zhang Y, Zhao L, Zhao W, Wang X, Ye E, et al. AFF4 facilitates melanoma cell progression by regulating c-Jun activity. Exp Cell Res. 2021;399:112445.

29. Cheng M, Sheng L, Gao Q, Xiong $Q$, Zhang H, Wu M, et al. The m(6)A methyltransferase METTL3 promotes bladder cancer progression via AFF4/NF-KB/MYC signaling network. Oncogene. 2019;38:3667-80.

30. Gao Q, Zheng J, Ni Z, Sun P, Yang C, Cheng M, et al. The m(6)A methylation-regulated AFF4 promotes self-renewal of bladder cancer stem cells. Stem Cells Int. 2020;2020:8849218.

31. Li J, Ge J, Yang Y, Liu B, Zheng M, Shi R. Long noncoding RNA ZFPM2-AS1 is involved in lung adenocarcinoma via miR-511-3p/AFF4 pathway. J Cell Biochem. 2020;121:2534-42.

\section{Publisher's Note}

Springer Nature remains neutral with regard to jurisdictional claims in published maps and institutional affiliations. 